\title{
THE DETERMINATION METHOD OF EXTREME EARTHQUAKE DISASTER AREA BASED ON THE DUST DETECTION RESULT FROM GF-4 DATA
}

\author{
Aixia Dou*, Ling Ding, Meng Chen, Xiaoqing Wang \\ Institute of Earthquake Forecasting, China Earthquake Administration, Beijing, China -axdothy @163.com
}

Commission VI, WG VI/4

KEY WORDS: Earthquake, Extreme Disaster Area, Dust, GF-4, Remote Sensing

\begin{abstract}
:
The remote sensing has played an important role in many earthquake emergencies by rapidly providing the building damage, road damage, landslide and other disaster information. The earthquake in the mountains often caused to the loosening of the mountains and the blowing of the dust in the epicentre area. The dust particles are more serious in the epicentre area than the other disaster area. Basis on the analysis of abnormal spectrum characteristics, the dust detection methods from medium and high resolutions satellite imagery are studied in order to determinate the extreme earthquake disaster area. The results indicate the distribution of extreme disaster can be acquired using the dust detection information from imagery, which can provide great help for disaster intensity assessment.
\end{abstract}

\section{INTRODUCTION}

After the serious destructive earthquake, the rapid impact acquisition of disaster information is crucial for the earthquake emergency response, emergency rescue and disaster loss assessment. Remote-sensing satellites can provide the important information about the affected areas. Remote-sensing technologies has been rapidly developed and play an important role in the process of emergency after Jiashi Xinjiang earthquake in 2003, Wenchuan earthquake in 2008, Yushu earthquake in 2010, Haiti earthquake in 2010, the Japanese earthquake and tsunami disaster in 2011, Nepal earthquake in 2015 etc. The main information extracted from images includes the building damage, road damage, landslide and other seismic geological hazards. However, the ground movement caused by the earthquake led to the loosening of the mountains and the blowing of the dust in the epicentre area. The dust particles are more serious in the epicentre area than the other disaster area, and they existed for long time in the air. The characteristics of dust particles affect the radioactive spectra directly through emissivity, particle size and refractive index. For this reason, the dust detection from the satellite is essential to determination the extreme earthquake disaster area. In this study, the approach of dust detection using remote sensing technology is presented and applied to the determination of the extreme disaster area after the earthquake.

\section{STUDY AREA AND DATASET}

\subsection{Study Area}

The earthquake with Ms 6.9 occurred in Milin, Tibet province at 6:34, November 182017 . The epicentre is located at $29.75^{\circ} \mathrm{N}$ and $95.02^{\circ} \mathrm{E}$, with focal depth 10 kilometres. This earthquake is located close to the north-eastern corner where the Indian plate underthrusts beneath the Eurasian plate. Here the crustal shortening and the tectonically rotated deformation are the strongest with respect to other areas along the whole Himalayan orogenic belt. The extreme disaster area was located in the mountain area with the high elevation of about 4000 meters above sea level. So it is difficult for investigators to get the disaster information in the field. The Fig. 1 shows the location and the topography of study area.

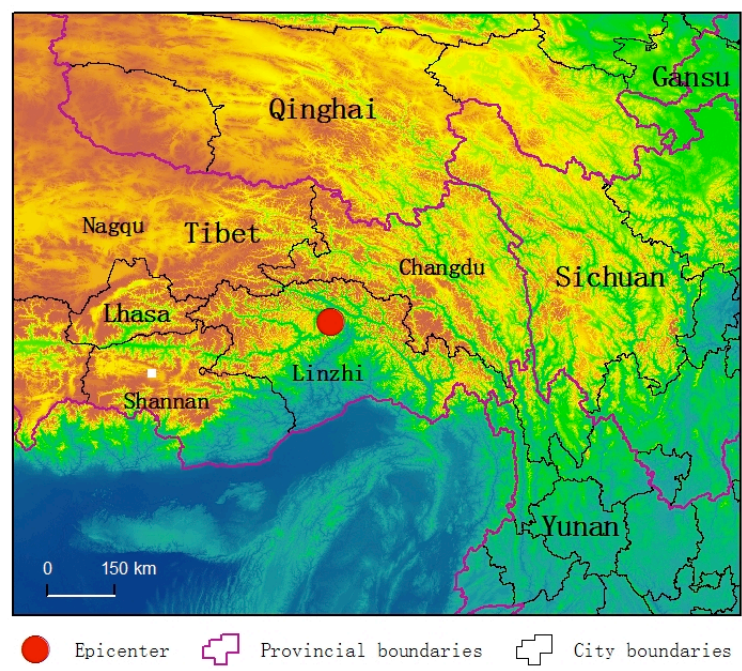

Figure 1. The location of study area

\subsection{Dataset}

The remote sensing images acquired by GaoFen-4 (GF-4) satellite from November 18th to 21 th, are used to detect the dust. GF-4 is the China's first stationary orbit satellite with the high resolution, which was launched in December 2015 and configured with a visible, near infrared (NIR) camera and an intermediate infrared camera, with the all-weather monitoring ability of visible and infrared bands. It can carry out the longterm continuous observation of some area of about $1 / 3$ of the earth's surface. The spectrum range of visible band is 0.45 $0.9 \mu \mathrm{m}$, with a resolution of 50 meters. The spectrum range of middle infrared band is $3.5-4.1 \mu \mathrm{m}$, with a resolution of 400 meters. The table 1 show the primary characteristics of

* Corresponding author 
panchromatic and multispectral sensor about GF-4 satellite. This satellite provided the first optical remote sensing data at 14:54 November 18 after earthquake. The images covering the whole disaster area are pre-processed. First, the apparent reflectivity is obtained by using the satellite parameters to radiometric calibration the image. Then the clouds are removed and the water is extracted.

\begin{tabular}{|c|c|c|c|c|c|}
\hline & $\begin{array}{l}\text { Spectrum } \\
\text { Band } \\
\text { NO. }\end{array}$ & $\begin{array}{l}\text { Spectral } \\
\text { Range }(\mu m)\end{array}$ & $\begin{array}{l}\text { Spectral } \\
\text { Resolution } \\
(m)\end{array}$ & $\begin{array}{l}\text { Swath } \\
(\mathrm{km})\end{array}$ & $\begin{array}{l}\text { Revisit } \\
\text { Period }\end{array}$ \\
\hline \multirow{5}{*}{ VNIR } & 1 & $0.45-0.90$ & \multirow{5}{*}{50} & \multirow{5}{*}{400} & \multirow{5}{*}{$20 \mathrm{~s}$} \\
\hline & 2 & $0.45-0.52$ & & & \\
\hline & 3 & $0.52-0.60$ & & & \\
\hline & 4 & $0.63-0.69$ & & & \\
\hline & 5 & $0.76-0.90$ & & & \\
\hline MWIR & 6 & $3.50-4.10$ & 400 & 400 & $20 \mathrm{~s}$ \\
\hline
\end{tabular}

Table 1. The parameters of GF-4

\section{ALGORITHM}

\subsection{Brightness normalization}

Before performing the analysis of the dust anomalous spectral characteristics, the spectral discrepancy of the same objects is taken into account and a brightness normalization method was applied to highlight spectral shape information and weaken the absolute reflectance. The normalized brightness value can be calculated by the equation (1):

$$
B_{i}^{k l}=\frac{n \times b_{i}^{k l}}{\sum_{j=1}^{n} b_{j}^{k l}}
$$

Where $b_{i}^{k l}$ is the pixel value of the $i$ band at the $k$ line, $l$ column, $n$ is the total number of bands.

The normalized spectrums of samples are presented in figure 2 . Compared with the original curves of spectrum (above picture), the internal variation of samples decreases a lot, which reduces the absolute reflectance and highlights the differences between different bands.
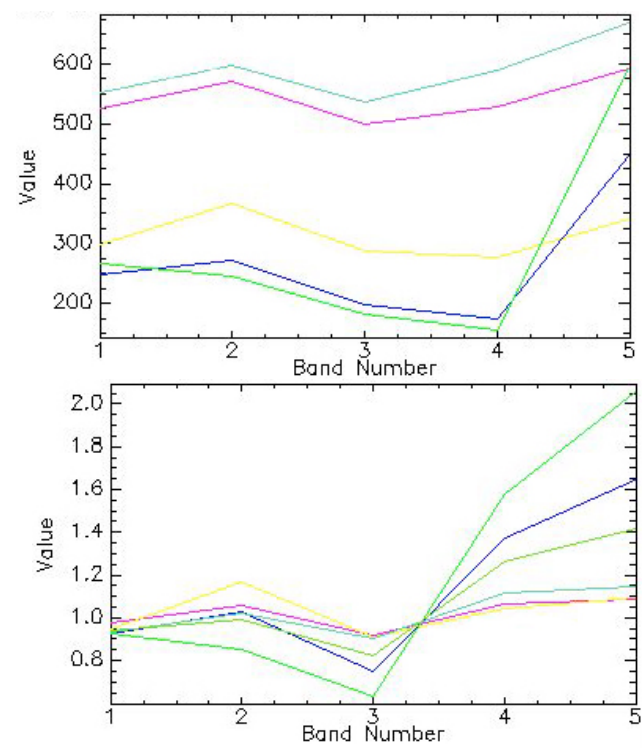

Figure 2. The mean of brightness before and after normalization

\subsection{Analysis of the spectrum abnormal features}

The samples are selected from the remote sensing images. They are the thick clouds, edge of thick clouds, thin clouds, and ground object near to the epicentre and in the other disaster area. These sample histograms of different bands and their mean, standard deviation are calculated, shown in the Fig.3. By comparing and analysing the spectral mean and histograms characteristics of four kinds of samples, the obvious characteristics of abnormal spectrum are shown. From the Fig.3, we can see that the spectrum of ground object is obviously difference from band 2 to band 5 . The brightness of band 2 and band 3 is higher in the area near to the epicentre than the others. However, the brightness of band 4 and band 5 is smaller in the area near to the epicentre than the others. The spectral characteristics of thin clouds are similar to the ground object. Because the thick clouds have strong reflections, the histograms of normalized brightness in both two areas have little difference. However, at the edge of thick clouds, the histogram presents two peaks in band 1 and band 2 in the area near to epicentre, but there is a peak far away from the epicentre. These characteristics mean that the dust changed the reflectivity of object in the area near to epicentre. So we can identify the extreme earthquake disaster area.

\section{DETECTION RESULTS AND VALIDATION}

On the basis of the analysis of the abnormal spectral features, and the dust distribution identified from images, combined with the field observations, the extreme earthquake disaster area is estimated and shown in Fig.4. The area is about 850 square kilometres. In order to assess the accuracy of this method, the spatial distribution of aftershocks and earthquake intensity of field investigation are used to verify the results. Figure 5 shows the earthquake scene pictures before and after Milin earthquake. Comparing the two pictures, we can see that before the earthquake, the sky is bright blue and the mountains are clear, but after the earthquake both the sky and the mountains are grey.

\section{SUMMARY AND CONCLUSION}

The validation result indicates that the spatial distribution of extreme disaster can be acquired comprehensively using medium and high resolutions satellite data. The GF-4 is an important data source of quickly identified the extreme disaster area, which can provide great help for disaster intensity assessment.

\section{ACKNOWLEDGEMENTS (OPTIONAL)}

This study was supported in part by National Key Research and Development program of China (2017YFB0504104). 

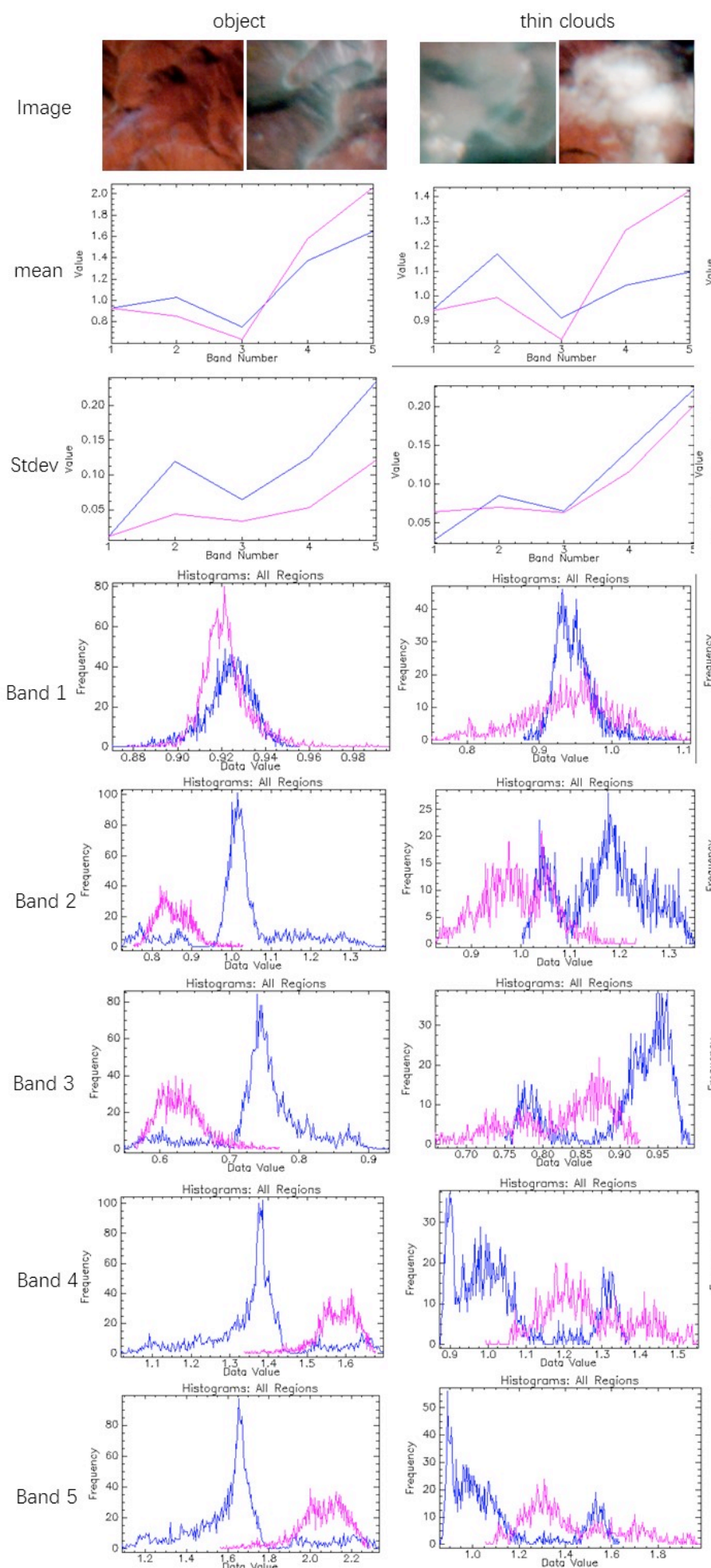

Figure 3. Spectrum mean, Stdev and histograms of different sample
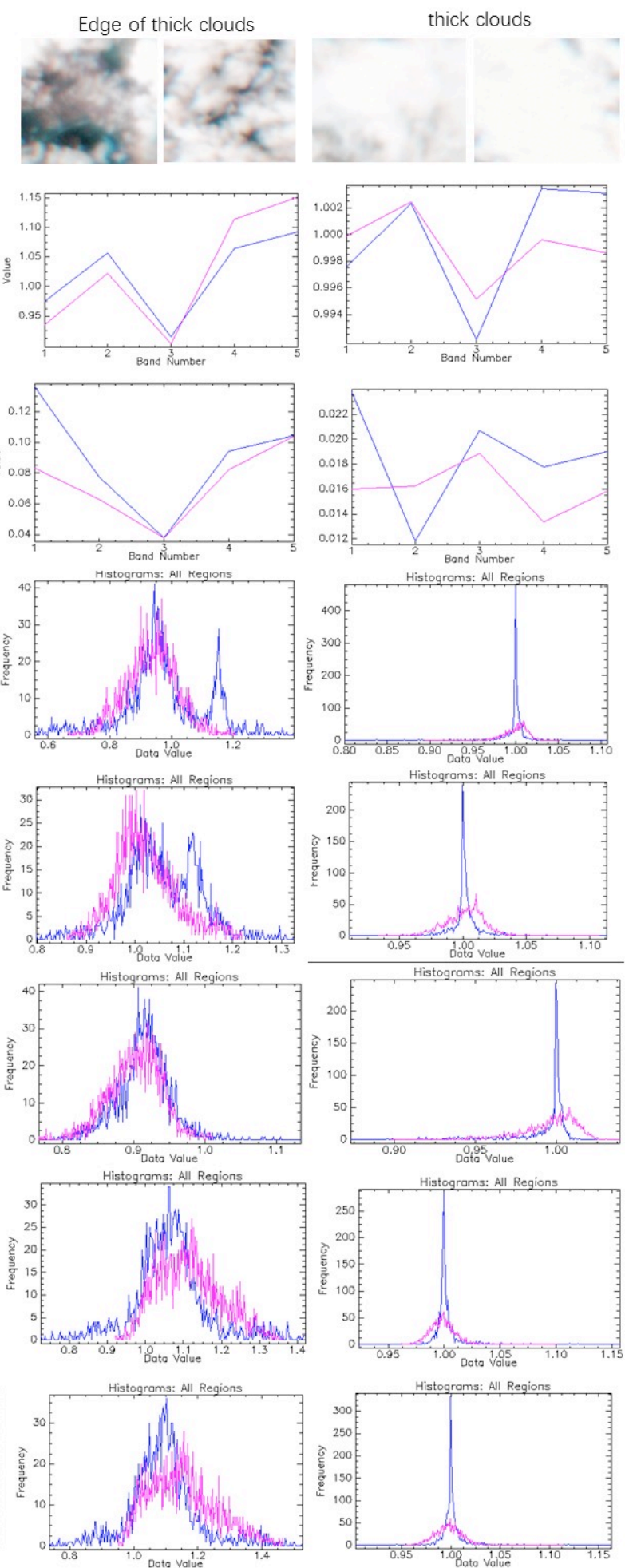

(The blue cuvees are the samples near to epicentre and the purple cuvees are samples in the other areas.) 


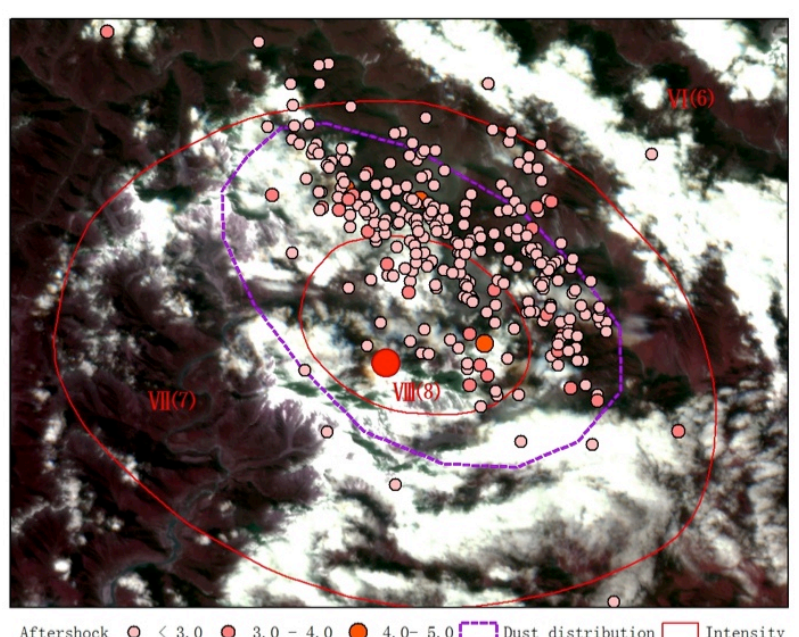

Figure 4. The dust distribution detected from GF-4
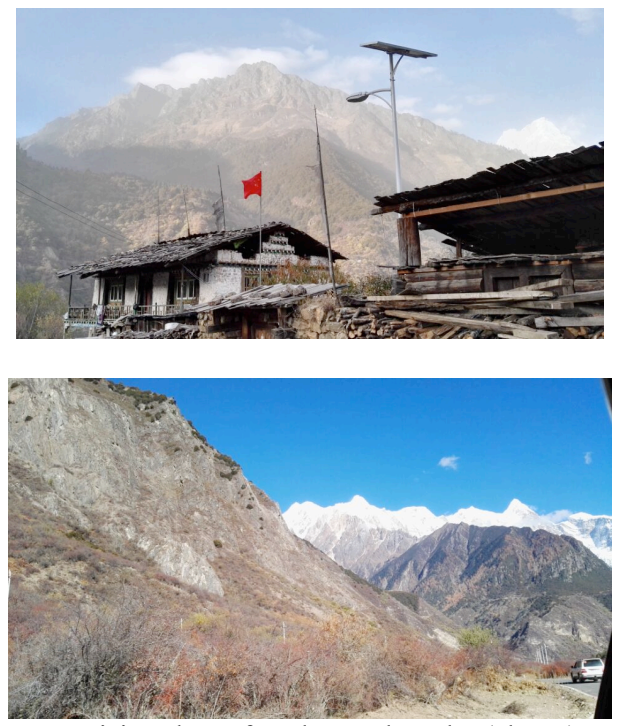

Figure 5. Raising dust after the earthquake (above) no dust (below)

\section{REFERENCES}

Bai, L., Li, G. H., Song, B. W., 2017.The source parameters of the M6.9 Mainling, Tibet earthquake and its tectonic implications. Chinese J. Geophys, 60(12), pp.4956-4963.

El-Askary, H.; Kafatos, M.; Liu, X.; et al., C. (2003):Introducing new approaches for dust storms detection using remote sensing technology In: Geoscience and Remote Sensing Symposium. IEEE International vol.4: 2439-2441.

Sang, S.P., Kim, J., Lee, J., et al., 2014. Combined dust detection algorithm by using MODIS infrared channels over East Asia. Remote Sensing of Environment, 141(2), pp. 24-39.

Wang, Z.T., Zhang, Y.H., Yuan, S.Y., et al., 2016.The aerosol monitoring over Beijing-Tianjin-Hebei region from GF-4 data. Environment and Sustainable development, 41(5), pp. 113-116.

Wang, Z.T., Xin, J. Y., Jia, S. L., Li, Q., Chen, L. F. and Zhao, S. H., 2015. Retrieval of AOD from GF-1 16 m camera via DDV algorithm. Journal of Remote Sensing, 19(3), pp. 530-538.

Xiong, W.C., Xu, Y.M., Li, J.R., et al. 2017.Urban dust pollution sources monitoring based on medium and high resolution satellite imagery in Tianjin. Remote sensing information. 32(3), pp.45-49.

Zhou, B., Zhou, N.Y., 2017.The Application of Remote Sensing in Dust Storm Detection. Geomatics \& Spatial Information Technology. 